\title{
Regadenoson in the detection of coronary artery disease
}

\author{
Christiane Buhr' \\ Mario Gössl ${ }^{2}$ \\ Raimund Erbel' \\ Holger Eggebrecht ${ }^{1}$ \\ 'Department of Cardiology, \\ West-German Heart Center Essen, \\ University of Duisburg-Essen, Essen, \\ Germany; ${ }^{2}$ Cardiovascular Diseases, \\ Mayo Clinic College of Medicine, \\ Rochester, MN 55905, USA
}

\begin{abstract}
Myocardial perfusion studies use either physical exercise or pharmacologic vasodilator stress to induce maximum myocardial hyperemia. Adenosine and dipyridamole are the most commonly used agents to induce coronary arterial vasodilation for myocardial perfusion imaging. Both cause frequent undesirable side-effects. Because of its ultrashort half-life, adenosine must be administered by constant intravenous infusion during the examination. A key feature of an ideal A2A agonist for myocardial perfusion imaging studies would be an optimal level and duration of hyperemic response. Drugs with a longer half-time and more selective A2A adenosine receptor agonism, such as regadenoson, should theoretically result in a similar degree of coronary vasodilation with fewer or less severe side-effects than non-selective, ultrashortlasting adenosine receptor stimulation. The available preclinical and clinical data suggest that regadenoson is a highly subtype-selective, potent, low-affinity A2A adenosine receptor agonist that holds promise for future use as a coronary vasodilator in myocardial perfusion imaging studies. Infusion of regadenoson achieves maximum coronary hyperemia that is equivalent to adenosine. After a single bolus infusion over $10 \mathrm{~s}$, hyperemia is maintained significantly longer (approximately $2-5 \mathrm{~min}$ ) than with adenosine, which should facilitate radionuclide distribution for myocardial perfusion imaging studies. In comparison with the clinically competitive A2A adenosine receptor agonist binodenoson, regadenoson has a several-fold shorter duration of action, although the magnitude of hyperemic response is comparable between the two. The more rapid termination of action of regadenoson points to an advantage of enhanced control for the clinical application. Regadenoson selectively causes vasodilation of the coronary circulation, whereas effects on systemic blood pressure are only mild. The clinical adverse effect profile of regadenoson appears to be favorable, particularly with respect to dreaded atrioventricular conduction disturbances and bronchospasm.
\end{abstract}

Keywords: regadenoson, adenosine, coronary vasodilation, physical exercise, ultrashort-lasting adenosine receptor stimulation, selective $\mathrm{A} 2 \mathrm{~A}$ adenosine receptor agonism

\section{Introduction}

Non-invasive imaging of relative regional myocardial perfusion by stress single photon emission computerized tomography (SPECT), magnetic resonance imaging (MRI), positron emission tomography (PET), or contrast-enhanced echocardiography plays an important role in the diagnosis of coronary artery disease (CAD) (Baghdasarian and Heller 2005). Myocardial perfusion studies use either physical exercise or pharmacologic vasodilator stress to induce maximum myocardial hyperemia. Adenosine and dipyridamole are the most commonly used agents to induce coronary arterial vasodilation for myocardial perfusion imaging. Both are given as a continuous infusion and act by a final common pathway: stimulating adenosine A2A receptors on arteriolar vascular smooth muscle cells. Unfortunately this effect is non-selective and there is activation of adenosine $\mathrm{A} 1, \mathrm{~A} 2 \mathrm{~B}$, and $\mathrm{A} 3$ receptors, which results in frequent undesirable side-effects such as chest pain and dyspnea, and less frequent but serious side-effects such as bronchospasm and high-grade atrioventricular (AV) 
block (Ranhosky and Kempthorne-Rawson 1990; Cerqueira et al 1994). In addition, hypotension resulting from adenosine-induced vasodilation in the peripheral circulation is another undesirable effect that limits the clinical usefulness of adenosine (Cerqueira 2004).

Because of its ultrashort half-life, adenosine must be administered by constant intravenous infusion during the examination. Bolus administration would obviate the need for an infusion pump, simplifying drug delivery during the examination. Another key feature of an ideal A2A agonist for myocardial perfusion imaging studies would be an optimal level and duration of hyperemic response. A rapid 2 - to 3-fold increase in coronary blood flow is required. In addition, hyperemia should be maintained to allow for adequate distribution of radionuclides within the myocardium (Cerqueira 2004). The time course of hyperemia should be as short as possible to minimize the risk of side-effects. On the other hand, hyperemia should be long enough (approximately 2-4 min) to allow maximal extraction of the radiotracer during the induced heterogeneous blood flow for detection of disparities caused by obstructive CAD (Cerqueira 2004).

Therefore, drugs with a longer half-life and more selective A2A adenosine receptor agonism should theoretically result in a similar degree of coronary vasodilation with fewer or less severe side-effects than non-selective, ultrashortlasting adenosine receptor stimulation. There is however a large A2A receptor "reserve" in the coronary artery, so that an ideal A2A agonist may well have a low affinity (Belardinelli et al 1996). Since the discovery of the prototypical A2A agonist CGS-21680 in 1989, several potent, selective A2A adenosine receptor agonists have been synthesized, and preliminary experimental studies have been promising. Currently, three selective adenosine A2A receptor agonists have begun phase III studies as pharmacologic stress agents, regadenoson (CVT3146; CV Therapeutics, Inc. and Astellas Pharma, Inc.), binodenoson (MRE0470 or WRC0470; King Pharmaceuticals R\&D Inc), and apadenoson (BMS068645 or ATL146e). This article reviews the preclinical and clinical studies of regadenoson with respect to its potential for myocardial perfusion imaging.

\section{Synthesis and structure activity relationship (SAR)}

Regadenoson is a 2-[N-1-(4-N-methylcarboxamidopyrazolyl)]-adenosine derivative. There are three biochemical classes of A2A adenosine receptor agonists based on two different 2-substituted adenosine derivatives. The first of these classes contains a pi system at the 2-position; for example, the acetylenic and trans double bonds of YT-146 (2-(1-octynyl)adenosine) and THENECA (2-(trans-1-hexenyl)adenosine), respectively, are used to extend a hydrophobic chain into a putative lipophilic pocket in the $\mathrm{A} 2 \mathrm{~A}$ receptor. A second class of $\mathrm{A} 2 \mathrm{~A}$ agonists contains a 2-heteroatom alkyl linker to a hydrophobic group (eg, CGS-21680). A third class of 2-substituted A2A adenosine agonists contains both a 2-heteroatom linker and a pi system (eg, binodenoson). Binodenoson has been demonstrated to be functionally selective for the A2A adenosine receptor, relative to the $\mathrm{A} 1$ receptor. Regadenoson has a $152^{\circ}$ trajectory, similarly to THENECA, but contains a 2-heteroatom linker and a pi system like that of binodenoson (Zablocki et al 2001). Previous research had demonstrated that 2-(3,5-disubstituted-N-pyrazolyl)-adenosine derivatives were inactive at doses of up to $100 \mu \mathrm{g}$ as coronary vasodilators, and further SAR investigation suggested that an extended presentation of the 4-substituted pyrazole would be preferred for selective binding at the $\mathrm{A} 2 \mathrm{~A}$ receptor (Palle et al 2000). During biochemical binding studies, regadenoson was found to be a short-acting coronary vasodilator $\left(\mathrm{t}_{1 / 2}=5.2 \pm 0.2 \mathrm{~min}\right)$ with good potency $\left(\mathrm{EC}_{50}=6.4 \pm 1.2 \mathrm{nM}\right)$ and functional selectivity for the $\mathrm{A} 2 \mathrm{~A}$ adenosine receptor versus the A1 receptor (Zablocki et al 2000).

Regadenoson was prepared as part of a series of 2-( $\mathrm{N}$ or C-pyrazolyl) adenosine derivatives including CVT-2995, CVT-3033, CVT-3032 and regadenoson, which, when characterized by radioligand binding and cAMP assays, exhibited $\mathrm{Ki}$ values at A2A and A1 adenosine receptors of 305 and 866 , 2895 and 5836, 13651 and 6350, and 1269 and $16460 \mathrm{nM}$, respectively. The decline in cAMP accumulation elicted in $\mathrm{PC}-12$ cells was inversely related $\left(\mathrm{R}^{2}=0.85\right)$ to the affinity of the agonist for the receptor (Gao et al 2000).

Regadenoson was synthesized from 2-hydrazinoadenosine in two steps. Ethoxycarbonylmalondialdehyde was condensed with 2-hydrazinoadenosine in a 1:1 mixture of ethanoic acid and methanol. The resulting 2-ester was then converted directly to the amide regadenoson by aminolysis with methylamine (Palle et al 2002). Regadenoson could also be synthesized from 2-chloro or 2-iodo adenosine derivatives (Palle et al 2000; Zablocki et al 2000).

\section{Clinical development}

Kerensky et al (2002) examined the effects of regadenoson on cerebral blood flow (CBF) velocity as assessed by Doppler flow wire in 36 patients undergoing clinically indicated coronary catheterization. Regadenoson (10-500 $\mu \mathrm{g})$ caused a rapid increase in $\mathrm{CBF}$ velocity that was near peak within 
30-40 s after peripheral intravenous bolus injection. The maximum peak to baseline $\mathrm{CBF}$ velocity ratio was $3.2 \pm 0.6$, and was similar to that obtained by intracoronary injection of adenosine $(3.2 \pm 0.5)$. The duration of CBF velocity augmentation (at least 2-fold increase) was dose dependent; at $300 \mu \mathrm{g}$ the duration was $4.0 \pm 4.9 \mathrm{~min}$ and at $500 \mu \mathrm{g}$ was $6.9 \pm 7.6 \mathrm{~min}$. At the maximal applied dose of $500 \mu \mathrm{g}$, the increase in heart rate was $18.7 \pm 4.0 \mathrm{bpm}$ and the maximal decrease in systolic blood pressure was $8.7 \pm 7.6 \mathrm{mmHg}$.

Hendel et al (2003) compared regadenoson and adenosine for myocardial perfusion imaging using stress-SPECT in 35 patients. Regadenoson was administered by a intravenous bolus injection of 400 or $500 \mu \mathrm{g}$. There were no differences in ischemia detection between regadenoson-SPECT and adenosine-SPECT, with a regional concordance for ischemia extent and severity of $86.3 \%$ and $83.4 \%$, respectively.

ADVANCE MPI 1 is a pivotal phase III, multi-national, randomized, double-blind trial that was initiated in October 2003 [www.clinicaltrials.gov]. In this multinational trial, involving 784 patients with coronary artery disease undergoing SPECT, patients were randomized to either adenosine SPECT or regadenoson SPECT. The trial was designed to compare the pharmacological stress SPECT images used to assess reversible perfusion defects and to compare the safety and tolerability of the two stress agents. In late 2005 it was reported that the primary endpoint of the study was met, suggesting that regadenoson SPECT was comparable with adenosine SPECT at detecting areas of the heart with reduced blood flow (CV Therapeutics, Inc. 2005a, b). The most common adverse events were headache, chest pain, shortness of breath, flushing and gastrointestinal discomfort (CV Therapeutics, Inc. 2005a). This preliminary information has to be interpreted with caution until the final results are published in scientific format.

\section{Side-effects and contraindications}

Hendel et al (2004) reported safety and tolerability of regadenoson for 36 patients. Although adverse events were reported rather frequently (in $72 \%$ of patients), these were mostly of minor clinical importance (96\%) and resolved within 15 minutes without treatment in $91 \%$ of cases. Chest discomfort, headache, and abdominal pain were the most common side-effects and were similar distributed between regadenoson low and high dose groups. Flushing, dyspnea, and dizziness were, however, more frequently encountered in the higher dose group. So far, AV conduction abnormalities or relevant bronchoconstriction have not been reported. Hemodynamic effects of regadenoson included a decrease in blood pressure $(-5.9 \pm 10.7 \mathrm{mmHg})$ and increase of heart rate $(+21.9 \pm 10.4 \mathrm{bpm})$ (Hendel et al 2004).

\section{Current opinion}

So far, the available preclinical and clinical data suggest that regadenoson is a highly subtype-selective, potent, lowaffinity A2A adenosine receptor agonist that holds promise for future use as a coronary vasodilator in myocardial

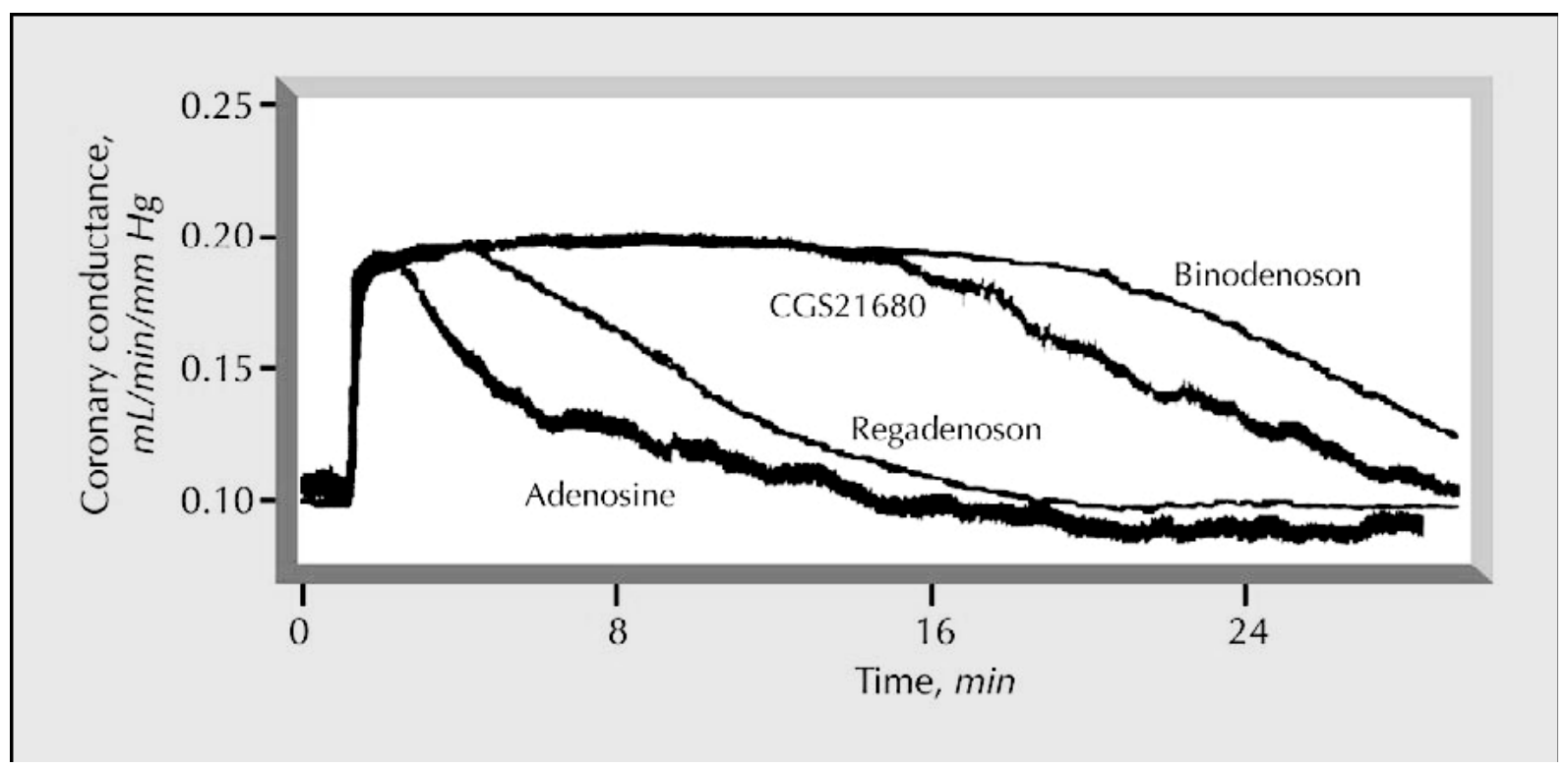

Figure I Time course of changes in coronary conductance caused by regadenoson, binodenoson, CGS2I680, and adenosine. Reprinted with permission from Gao Z, Otero $\mathrm{DH}$, Zablocki JA, et al 2000. Pharmacological characterization of novel A2A adenosine receptor (A2AAdoR) agonists. Drug Dev Res, 50:93. Copyright@ Wiley-Liss, Inc., a subsidiary of John Wiley \& Sons, Inc. 
perfusion imaging studies. Peripheral intravenous infusion of a regadenoson achieves maximum coronary hyperemia equivalent to that achieved with adenosine. After a single bolus infusion over $10 \mathrm{~s}$, hyperemia is maintained significantly longer (approximately 2-5 $\mathrm{min}$ ) than with adenosine, which should facilitate radionuclide distribution for myocardial perfusion imaging studies. In comparison with the clinically competitive A2A adenosine receptor agonist binodenoson, regadenoson has a several-fold shorter duration of action, although the magnitude of hyperemic response is comparable between the two.

The more rapid termination of action of regadenoson points to an advantage of enhanced control for the clinical application. As a low-affinity A2A receptor agonist, regadenoson selectively causes vasodilation of the coronary circulation, whereas effects on systemic blood pressure are only mild. According to its functional selectivity on the A2A adenosine receptor, AV conduction abnormalities or bronchoconstriction were not observed in preclinical studies.

Preliminary clinical data suggest that a bolus of $400 \mu \mathrm{g}$ administered by peripheral intravenous injection is sufficient to achieve maximum coronary hyperemia that lasts for approximately 4-7 $\mathrm{min}$. The clinical adverse effect profile of regadenoson appears to be favorable, particularly with respect to the dreaded $\mathrm{AV}$ conduction disturbances and bronchospasm; however, the current side-effect profile of regadenoson is based on only small populations of patients. Therefore, larger-scale studies are mandatory to reliably establish the clinical safety of regadenoson and its usefulness for myocardial perfusion imaging studies.

\section{References}

Baghdasarian SB, Heller GV. 2005. The role of myocardial perfusion imaging in the diagnosis of patients with coronary artery disease: developments over the past year. Curr Opin Cardiol, 20:369-74.
Belardinelli L, Shryock JC, Ruble J, et al. 1996. Binding of the novel nonxanthine A2A adenosine receptor antagonist [3H]SCH58261 to coronary artery membranes. Circ Res, 79:1153-60.

Cerqueira MD. 2004. The future of pharmacologic stress: selective A2A adenosine receptor agonists. Am J Cardiol, 94:33D-40D (472226).

Cerqueira MD, Verani MS, Schwaiger M, et al. 1994. Safety profile of adenosine stress perfusion imaging: results from the Adenoscan Multicenter Trial Registry. J Am Coll Cardiol, 23:384-9.

CV Therapeutics, Inc. 2005a. Astellas and CV's regadenoson comparable to Adenoscan in heart imaging study [press release] August 10, 2005.

CV Therapeutics, Inc. 2005b. Regadenoson Phase 3 Data Presented as LateBreaker at American Society of Nuclear Cardiology [press release]. October 2, 2005.

Hendel RC, Mahmarian JJ, Bateman TM, et al. 2004. Initial results regarding safety, tolerability, and hemodynamic effects of CVT-3146, a Selective Adenosine $\mathrm{A} 2 \mathrm{~A}$ receptor agonist, in patients undergoing pharmacologic stress SPECT myocardial perfusion imaging [abstract]. J Am Coll Cardiol, 43:367A.

Hendel RC, Mahmarian JJ, Cerqueira MD et al. 2003. Pharmocologic stress SPECT myocardial perfusion imaging with a selective A2A agonist: results of a pilot study comparing adenosine with CVT-3146 [abstract]. Circulation, 108:17.

Gao Z, Otero DH, Zablocki JA, et al. 2000. Pharmacological characterization of novel A2A adenosine receptor (A2AAdoR) agonists. Drug Dev Res, 50:93.

Kerensky RA, von Mering GO, Kent KM, et al. 2002. Dose dependent increase in human coronary blood flow velocity after an iv bolus of CVT-3146, a novel A2A adenosine receptor agonist: a potential agent for the use in pharmacological stress testing for myocardial perfusion imaging [abstract]. Circulation, 106:618.

Palle V, Elzein E, Gothe SA, et al. 2000. Design, synthesis and pharmacological evaluation of 2(1-alkyl-pyrazol-4-yl) adenosine derivatives as short acting adenosine A2A receptor agonists. Drug Dev Research, 50:64.

Palle VP, Elzein EO, Gothe SA, et al. 2002. Structure-affinity relationships of the affinity of 2-pyrazolyl adenosine analogues for the adenosine A2A receptor. Bioorg Med Chem Lett, 12:2935-9.

Ranhosky A, Kempthorne-Rawson J. 1990. The safety of intravenous dipyridamole thallium myocardial perfusion imaging. Intravenous Dipyridamole Thallium Imaging Study Group. Circulation, 81:1205-9.

Zablocki J, Palle V, Blackburn B, et al. 2000. Novel short acting coronary vasodilators that are functionally selective for the A2A receptor based on 2-heterocyclic substituted adenosine derivatives. Drug Dev Res, 50:63.

Zablocki J, Palle V, Blackburn B, et al. 2001. 2-substituted pi system derivatives of adenosine that are coronary vasodilators acting via the A2A adenosine receptor. Nucleosides Nucleotides Nucleic Acids, 20:343-60. 